\title{
Belphégor
}

\section{Paola Irene Galli Mastrodonato e Maria Gabriella Dionisi (a cura di), Riletture salgariane}

\section{Fabiana Dimpflmeier}

\section{Q OpenEdition}

1 Journals

\section{Edizione digitale}

URL: http://journals.openedition.org/belphegor/502

DOI: 10.4000/belphegor.502

ISSN: 1499-7185

Editore

LPCM

\section{Notizia bibliografica digitale}

Fabiana Dimpflmeier, «Paola Irene Galli Mastrodonato e Maria Gabriella Dionisi (a cura di), Riletture salgariane », Belphégor [En ligne], 12-1 | 2014, mis en ligne le 12 juin 2014, consulté le 22 septembre 2020. URL : http://journals.openedition.org/belphegor/502 ; DOI : https://doi.org/10.4000/belphegor. 502

\section{Questo documento è stato generato automaticamente il 22 settembre 2020.}

\section{c) (i) $\Theta$}

Belphégor est mis à disposition selon les termes de la Licence Creative Commons Attribution - Pas d'Utilisation Commerciale - Pas de Modification 4.0 International. 


\section{Paola Irene Galli Mastrodonato e Maria Gabriella Dionisi (a cura di), Riletture salgariane}

Fabiana Dimpflmeier

NOTIZIA

Paola Irene Galli Mastrodonato e Maria Gabriella Dionisi (a cura di), Riletture salgariane, Pesaro, Metauro Edizioni S.r.l., 2012, pp. 297, ISBN 9788861560802 
1 Riletture salgariane - edito sulla scia delle numerose pubblicazioni comparse in occasione del Centenario della morte (21 agosto 1911) di Emilio Salgari - già dal titolo si propone come una rivisitazione matura, 'plurale' e 'polifonica' dell'opera del Nostro, a partire dall'analisi dei testi fino ad arrivare alla loro ricezione e influenza sulla contemporaneità. I punti di vista si moltiplicano, si intrecciano, a volte convergendo e altre volte correndo paralleli, ad impreziosire, nella molteplicità degli sguardi, il composito corpus salgariano.

Nell'insieme il volume punta a sottolineare la necessità di ulteriori ampliamenti delle ricerche nel senso di una differenziazione fra gli scritti più conosciuti (e maggiormente studiati) e la produzione minore dell'autore che serva

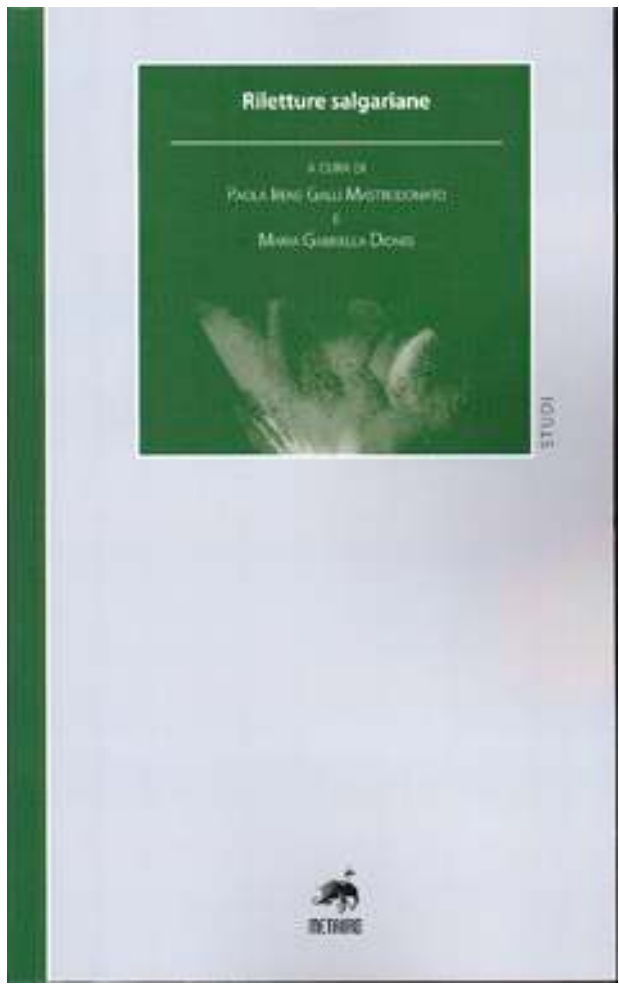
ad esaltarne il rispettivo peso e posizionamento all'interno dell'opera, nonché verso nuovi e prolifici approfondimenti o contaminazioni suggestive (e penso, fra gli altri, allo squisito saggio di Mario Tropea, alle osservazioni di Rosa Maria Grillo e alle provocazioni di Corrado Farina).

3 Significativo è il tentativo di concentrare l'attenzione sul rapporto fra Salgari e il panorama di immagini, fonti e notizie contemporanee che possono averne influenzato il processo compositivo, secondo un'ottica che punta ad una esegesi critica del loro utilizzo che trascende il testo per concentrarsi sulla rappresentatività e/o originalità nel contesto coevo, aprendo un intero capitolo di indagine sulle rappresentazioni dell'alterità utilizzate dallo scrittore veronese.

Così si passa dagli scenari salgariani intesi come “"luoghi dell'oggi' [...] dove soffia il vento della Storia" (p.15) di Paola Irene Galli Mastrodonato e le dotte riflessioni di Felice Pozzo - che ribadisce che "un sottofondo storico esiste e costituisce anzi una peculiarità dell'opera di Salgari, che ha romanzato, spesso in tempo reale, numerosi avvenimenti del colonialismo europeo" (p. 83-84) - al rapporto tra politica e poetica (Claudio Gallo), realismo e dimensione fantastica (Alfredo Luzi, Maria Gabriella Dionisi); per arrivare alla più sottile questione dell'orientalismo di (e in) Salgari, affrontato più diffusamente da Michelguglielmo Torri e Maria Luisa Longo.

5 La parte dedicata al Sud America sembra aprire a nuovi scenari e rappresentazioni che accrescono la geografia e l'antropologia dello scrittore e le sue possibilità interpretative. Claudia Borri, attraverso una ricca e puntuale peregrinazione fra le popolazioni indigene sudamericane, arriva a concepire la funzione del 'selvaggio' salgariano come il riflesso di "un sentire comune che affonda le sue radici nelle cronache della conquista, secondo il quale agli antipodi vivono popoli primitivi e selvaggi, simili, per molti versi, agli animali" (p. 144). Non diversamente Ilaria Magnani, che parla di "risignificazione interpretativa ottocentesca e positivista" dei topoi del 
medioevo europeo, mentre Nicola Bottiglieri sottolinea quanto dai luoghi comuni salgariani fatti di una "solida geografia emozionale" e di "un'antropologia fantasiosa" si evidenzi un immaginario da Italietta umbertina, fitto di trame da melodramma romantico.

6 Nell'insieme, mi sembra che il mondo di Salgari, pur aprendo possibilità di azione potenzialmente irrealizzabili nella sua realtà, non sconfini dal panorama immaginativo e storico contemporaneo. I suoi personaggi, come le ambientazioni, prendono vita a partire da uno sfondo erudito di conoscenze che si sviluppa secondo mappature e potenzialità prettamente eurocentriche.

7 Va forse allora ricordato che la classificazione del mondo utilizzata dallo scrittore, per quanto ricca e variegata, botanicamente e zoologicamente precisa ed accurata, 'nobilmente' enciclopedica, è una forma di com-prensione e controllo della realtà che si è sviluppata, in forme diverse, sin dalla scoperta dell'America. Non esiste sapere libero da costrizioni ideologiche e logiche di potere: non sono le fonti di Salgari ad essere razziste; è la modalità stessa di rappresentazione dell'alterità del periodo che non lascia spazio all'esistenza di modalità alternative dell'essere. La modernità si è costruita attraverso un regime discorsivo basato sul rapporto fra centro e periferie creato come supporto ideologico e politico alla dominazione sull'altro: un discorso coloniale che ha prodotto la realtà degli oggetti che rappresenta e dei soggetti che se ne fanno latori. L'identità occidentale e la modernità stessa non possono essere capite né pensate se non in relazione alla costruzione di un altro esotico, primitivo, selvaggio o barbaro (Bhabha, Spivak, Barker 1984; Said 1998; Bhabha 2001).

8 La novità di Salgari, a mio parere, sta piuttosto nel far muovere personaggi 'ai margini' secondo valori europei, nel far passare la sua erudizione, il suo modo di viaggiare attraverso le fonti, attraverso un processo di rielaborazione e ripensamento che porta alla personale creazione di un universo parallelo in cui i valori derivati dalla tradizione risorgimentale nostrana possono trovare spazio d'azione. Nulla a che vedere, però, con il riconoscimento dell'alterità. Al contrario, i suoi eroi (o anti-eroi) agiscono secondo i dettami delle rappresentazioni esotiche e orientaliste del tempo, completamente svuotati della loro essenza e/o successivamente addomesticati secondo i nostri valori (secondo un doppio meccanismo di negazione). Nella fantasia salgariana avviene un fagocitamento dell'altro che porta alla sua riduzione a presenza simbolica.

9 Come ricorda Grillo, citando Guido Sgardoli, l'esotico è "quel territorio dell'apparentemente lontano [...] è l'inattuale, un distante che scopriamo vicino, secondo il noto paradosso del romanzo, che ci parla di noi, del nostro mondo, delle cose e del tempo che in realtà conosciamo, ma non immediatamente riconoscibile..." (p. 279).

10 Ciò che forse differenzia - ai nostri occhi - lo scrittore italiano dai colleghi contemporanei inglesi e francesi e che lo rende apparentemente meno razzista e colonialista, è l'utilizzo di un immaginario formatosi quasi esclusivamente di riflesso: un 'discorso coloniale' - "inteso come insieme di atteggiamenti e di comportamenti, di mentalità e di miti ereditati da secoli di dominio coloniale" (Labanca 2002) caratterizzato ancora poco da una strumentalizzazione propagandistica forte e da una rigida stereotipizzazione. Un bacino di immagini con cui era facile giocare, e che conteneva i germi, le crepe e le potenzialità di molti dei discorsi sviluppatesi nei successivi cento anni di storia. Un sostrato che ancora oggi ci lega a Salgari e che determina il successo delle sue opere. 


\section{AUTORI}

\section{FABIANA DIMPFLMEIER}

Università di Roma 'La Sapienza' 